\title{
Fast algorithm for discrete fractional Hadamard transform
}

\author{
Aleksandr Cariow • Dorota Majorkowska-Mech
}

Received: 18 July 2013 / Accepted: 15 April 2014 / Published online: 15 August 2014

(C) Springer Science+Business Media New York 2014

\begin{abstract}
This paper proposes a fast algorithm for computing the discrete fractional Hadamard transform for the input vector of length $N$, being a power of two. A direct calculation of the discrete fractional Hadamard transform requires $N^{2}$ real multiplications, while in our algorithm the number of real multiplications is reduced to $N \log _{2} N$.
\end{abstract}

Keywords Discrete linear transforms - Discrete fractional Hadamard transform • Eigenvalue decomposition $\cdot$ Fast algorithms

\section{Introduction}

Discrete fractional Hadamard transform (DFRHT) is a generalization of the discrete Hadamard transform (DHT) with one additional fractional parameter as a discrete fractional Fourier transform is a generalization of the discrete Fourier transform. Since the fractional Fourier transform has been widely applied in the compression of signals [1], image encryption [2-4], digital watermarking [5] other fractional discrete orthogonal transforms were developed, such as: discrete fractional cosine and sine transforms [6] and the discrete fractional Hartley transform [7]. In [8] a discrete fractional Hadamard transform for the vector of length $N=2^{n}$ was defined, but a fast algorithm for the realization of this transform has not been proposed. The authors' paper [9] describes a rationalized algorithm for DFRHT, which possesses a reduced number of multiplications and additions. The analysis of the mentioned algorithm shows that not all of the existing improvement possibilities have been found. In

A. Cariow $(\triangle) \cdot$ D. Majorkowska-Mech

Faculty of Computer Science and Information Technology,

West Pomeranian University of Technology, Szczecin, Poland

e-mail: atariov@wi.zut.edu.pl 
this paper, we propose another algorithm for the discrete fractional Hadamard transform that requires fewer total real additions and multiplications, than our previously published solution.

\section{Mathematical background}

Hadamard matrices are defined as $N \times N$ symmetric matrices whose entries are either 1 or -1 and whose distinct rows are mutually orthogonal [10]. The normalized forms are denoted by $\mathbf{H}_{N}$, and for $N=2^{n}$ they can be recursively constructed as follows:

$$
\mathbf{H}_{2}=\frac{1}{\sqrt{2}}\left[\begin{array}{cc}
1 & 1 \\
1 & -1
\end{array}\right], \mathbf{H}_{N}=\frac{1}{\sqrt{2}}\left[\begin{array}{cc}
\mathbf{H}_{\frac{N}{2}} & \mathbf{H}_{\frac{N}{2}} \\
\mathbf{H}_{\frac{N}{2}} & -\mathbf{H}_{\frac{N}{2}}
\end{array}\right]
$$

for $N=4,8, \ldots, 2^{n}$. Matrices generated in this fashion (without normalization) were first constructed by James Joseph Sylvester [10]. They are referred to as Sylvester Hadamard matrices, but we will call them Hadamard matrices. These matrices have many interesting properties $[11,12]$ and are widely used in communication systems, data compression, error control coding, cryptography, linear filtering and spectral analysis $[13,14]$.

Definition of the discrete fractional Hadamard transform is based on eigenvalue decomposition of the DHT matrix. According to the Spectral Theorem, any real symmetric matrix (including the Hadamard matrix) can be written as a product [15]

$$
\mathbf{H}_{N}=\mathbf{Z}_{N} \Lambda_{N} \mathbf{Z}_{N}^{T}=\sum_{k=0}^{N-1} \lambda_{k} \mathbf{z}_{N}^{k}\left(\mathbf{z}_{N}^{k}\right)^{T}
$$

where $\Lambda_{N}$ is a diagonal matrix of order $2^{n}$, whose diagonal entries are the eigenvalues of $\mathbf{H}_{N}$

$$
\Lambda_{N}=\left[\begin{array}{cccc}
\lambda_{0} & & & \\
& \lambda_{1} & & \mathbf{0} \\
& 0 & \ddots & \\
& & & \lambda_{N-1}
\end{array}\right]
$$

$\mathbf{Z}_{N}=\left[\mathbf{z}_{N}^{0}\left|\mathbf{z}_{N}^{1}\right| \ldots \mid \mathbf{z}_{N}^{N-1}\right]$ - the matrix whose columns are normalized mutually orthogonal eigenvectors of the matrix $\mathbf{H}_{N}$. The eigenvector $\mathbf{z}_{N}^{k}$ corresponds to the eigenvalue $\lambda_{k}$. A superscript ${ }^{T}$ denotes the matrix transposition operation. The set of eigenvalues $\lambda_{0}, \lambda_{1}, \ldots, \lambda_{N-1}$ is called the spectrum of matrix $\mathbf{H}_{N}$. However, eigenvectors $\mathbf{v}_{N}^{k}$, which are columns of the matrix $\mathbf{Z}_{N}$ (after normalization), as well as their associated eigenvalues $\lambda_{k}$, can be ordered in different ways. In many cases, including the case of discrete fractional Hadamard transform, the so-called sequency ordering of the eigenvectors is used. This means that the $k$-th eigenvector has $k$ sign-changes. We will first clarify what is meant by a sign-change in a vector. The vector $\mathbf{v}_{N}=\left[v_{0}, v_{1}, \ldots, v_{N-1}\right]^{T}$ has a sign-change at $n$ if $v_{n} v_{n+1}<0$ for $n=0,1, \ldots, N-2$ or if $v_{n}=0$ and $v_{n-1} v_{n+1}<0$ for $n=1,2, \ldots, N-2$. Functions are the continuous counterparts of vectors. The sign-change of a vector 
corresponds to a "zero-crossing" of a function. The zero-crossing is a point where the sign of a function changes (e.g. from positive to negative or vice versa), and it is represented by a crossing of the horizontal axis (zero value) in the graph of the function. The bases of trigonometric functions, Walsh functions and orthogonal polynomials are indexed in such a way that the function possessing an index 0 has no zero-crossing, the function with an index 1 has one zero-crossing and so on. It can be said that $k$-th function has $k$ zero-crossings. The numbers of zero-crossings of the basis functions $f_{0}, f_{1}, f_{2}, \ldots$ create a sequence $0,1,2, \ldots$ It could be said that the functions are sequency ordered. Also the Hermite-Gaussian functions, being eigenfunctions of the Fourier transform, are sequency ordered. The discrete HermiteGaussians, the eigenvectors of the discrete Fourier transform, are ordered in this way as well [16]. The columns (rows) of the Hadamard matrix $\mathbf{H}_{N}$, defined by (1), are not sequency ordered (for $N \geq 4$ ), but the numbers of sign-changes appearing in these columns (rows) create the set $\{0,1, \ldots, N-1\}$ in some order. This means that the Hadamard matrix has a full column and row sign spectrum [12] and we can interchange the columns (rows) of this matrix, so as they were sequency ordered.

The discrete fractional Hadamard transform matrix of order $N=2^{n}$ with a real parameter $\alpha$ was first defined in [8]. This matrix is simply a power of the DHT matrix, where the exponent $a=\alpha / \pi$

$$
\mathbf{H}_{N}^{a}=\mathbf{Z}_{N} \Lambda_{N}^{a} \mathbf{Z}_{N}^{T}=\sum_{k=0}^{N-1} \lambda_{k}^{a} \mathbf{z}_{N}^{k}\left(\mathbf{z}_{N}^{k}\right)^{T}
$$

Obviously, for $a=0$ the DFRHT matrix becomes the identity matrix, and for $a=1$ it is transformed into ordinary DHT matrix. Generally a DFRHT matrix is complexvalued.

In order to obtain the discrete fractional Hadamard matrix defined by (4) it is necessary to calculate the eigenvalues and eigenvectors of the matrix $\mathbf{H}_{N}$. A method for finding the eigenvalues and eigenvectors of the Hadamard matrix has been presented in [17]. In turn, in [8] a recursive method for the calculation of the eigenvectors of the Hadamard matrix of order $2^{n+1}$ based on the eigenvectors of the Hadamard matrix of order $2^{n}$ has been proposed. In this article, this method will be stated briefly.

In [17] it was shown that if $\mathbf{v}_{N}^{k}(k=0,1, \ldots, N-1)$ is an eigenvector of the Hadamard matrix of order $N=2^{n}$ associated with an eigenvalue $\lambda$, then vector

$$
\hat{\mathbf{v}}_{2 N}^{k}=\left[\begin{array}{c}
\mathbf{v}_{N}^{k} \\
(\sqrt{2}-1) \mathbf{v}_{N}^{k}
\end{array}\right]
$$

will be an eigenvector of the matrix $\mathbf{H}_{2 N}$ associated with the eigenvalue $\lambda$.

In [8] it was shown that if $\mathbf{v}_{N}^{k}$ is an eigenvector of $\mathbf{H}_{N}$ associated with an eigenvalue $\lambda$, then vector

$$
\tilde{\mathbf{v}}_{2 N}^{k}=\left[\begin{array}{c}
(1-\sqrt{2}) \mathbf{v}_{N}^{k} \\
\mathbf{v}_{N}^{k}
\end{array}\right]
$$

will be an eigenvector of the matrix $\mathbf{H}_{2 N}$ associated with the eigenvalue $-\lambda$. 
These two results allow us to generate the eigenvectors of order $2^{n+1}$ from the eigenvectors of order $2^{n}$. Knowing the straightforward eigenvectors of the matrix $\mathbf{H}_{2}$

$$
\mathbf{v}_{2}^{0}=\left[\begin{array}{c}
1 \\
\sqrt{2}-1
\end{array}\right] \mathbf{v}_{2}^{1}=\left[\begin{array}{c}
1-\sqrt{2} \\
1
\end{array}\right]
$$

associated with the eigenvalues 1 and -1 respectively, the eigenvectors of the Hadamard matrix of arbitrary order $N=2^{n}$ can be recursively computed.

The only eigenvalues of the unnormalized Hadamard matrix of order $N=2^{n}$ are known to be $2^{n / 2}$ and $-2^{n / 2}$ [18], hence the normalized Hadamard matrix $\mathbf{H}_{N}$ has only the eigenvalues 1 and -1 , each one has an eigenspace of dimension $N / 2$. In [8] it was shown that the recursively computed eigenvectors with the formulas (5)-(7) are orthogonal and that the $2 N$ eigenvectors of $\mathbf{H}_{2 N}$ can be ordered in such a way that $\mathbf{v}_{2 N}^{k}$ has $k$ sign-changes. We illustrate the procedure with the example below.

Example 1 The number of sign-changes in eigenvectors $\mathbf{v}_{2}^{0}$ and $\mathbf{v}_{2}^{1}$ of matrix $\mathbf{H}_{2}$, determined by (7), is equal to 0 and 1 respectively. Using the expressions (5) and (6) we get the eigenvectors of matrix $\mathbf{H}_{4}$ :

$$
\hat{\mathbf{v}}_{4}^{0}=\left[\begin{array}{c}
1 \\
b \\
b \\
b^{2}
\end{array}\right] \quad \tilde{\mathbf{v}}_{4}^{0}=\left[\begin{array}{c}
-b \\
-b^{2} \\
1 \\
b
\end{array}\right] \quad \hat{\mathbf{v}}_{4}^{1}=\left[\begin{array}{c}
-b \\
1 \\
-b^{2} \\
b
\end{array}\right] \quad \tilde{\mathbf{v}}_{4}^{1}=\left[\begin{array}{c}
b^{2} \\
-b \\
-b \\
1
\end{array}\right],
$$

where $b=\sqrt{2}-1$. We note that the numbers of sign-changes in the above vectors are $0,1,3,2$ respectively $(b>0)$. Therefore, to obtain a sequency ordered set of eigenvectors of matrix $\mathbf{H}_{4}$, they should be numbered in the following manner:

$$
\mathbf{v}_{4}^{0}=\hat{\mathbf{v}}_{4}^{0} \quad \mathbf{v}_{4}^{1}=\tilde{\mathbf{v}}_{4}^{0} \quad \mathbf{v}_{4}^{2}=\tilde{\mathbf{v}}_{4}^{1} \quad \mathbf{v}_{4}^{3}=\hat{\mathbf{v}}_{4}^{1} .
$$

The corresponding eigenvalues will be equal to:

$$
\lambda_{0}=1 \quad \lambda_{1}=-1 \quad \lambda_{2}=1 \quad \lambda_{3}=-1 .
$$

It is easy to check that for $N=8$ the sequency ordered eigenvectors of matrix $\mathbf{H}_{8}$ will have the form:

$$
\begin{array}{ccccc}
\mathbf{v}_{8}^{0}=\hat{\mathbf{v}}_{8}^{0} & \mathbf{v}_{8}^{1}=\tilde{\mathbf{v}}_{8}^{0} & \mathbf{v}_{8}^{2}=\tilde{\mathbf{v}}_{8}^{1} & \mathbf{v}_{8}^{3}=\hat{\mathbf{v}}_{8}^{1} \\
\mathbf{v}_{8}^{4}=\hat{\mathbf{v}}_{8}^{2} & \mathbf{v}_{8}^{5}=\tilde{\mathbf{v}}_{8}^{2} & \mathbf{v}_{8}^{6}=\tilde{\mathbf{v}}_{8}^{3} & \mathbf{v}_{8}^{7}=\hat{\mathbf{v}}_{8}^{3},
\end{array}
$$

and the corresponding eigenvalues are equal to:

$$
\lambda_{0}=\lambda_{2}=\lambda_{4}=\lambda_{6}=1 \quad \lambda_{1}=\lambda_{3}=\lambda_{5}=\lambda_{7}=-1 .
$$

The relations obtained in Example 1 can be easily generalized as follows:

$$
\left\{\begin{array}{l}
\mathbf{v}_{2 N}^{4 l}=\hat{\mathbf{v}}_{2 N}^{2 l} \\
\mathbf{v}_{2 N}^{4 l+1}=\tilde{\mathbf{v}}_{2 N}^{2 l} \\
\mathbf{v}_{2 N}^{4 l+2}=\tilde{\mathbf{v}}_{2 N}^{2 l+1} \\
\mathbf{v}_{2 N}^{4 l+3}=\hat{\mathbf{v}}_{2 N}^{2 l+1}
\end{array}\right.
$$


for $l=0,1, \ldots, \frac{N}{2}-1$ and

$$
\lambda_{k}=(-1)^{k}
$$

for $k=0,1, \ldots, 2 N-1$.

It must be noted that both the eigenvectors of the matrix $\mathbf{H}_{2}$ and eigenvectors obtained for the Hadamard matrices of higher order are not normalized. In this article it is assumed that the notation $\left\|\mathbf{v}_{N}^{k}\right\|$ means the Euclidean norm of vector $\mathbf{v}_{N}^{k}$. In [9] it was shown that for any $N=2^{n}$ we have the relationship

$$
\left\|\mathbf{v}_{N}^{k}\right\|^{2}=\left(1+b^{2}\right)^{n}
$$

where $k=0,1, \ldots, N-1$ and $b=\sqrt{2}-1$. If we take the designation $c=1+b^{2}$, then normalized eigenvectors of the Hadamard matrix of order $N=2^{n}$ will take the form

$$
\mathbf{z}_{N}^{k}=\frac{\mathbf{v}_{N}^{k}}{\left\|\mathbf{v}_{N}^{k}\right\|}=\frac{\mathbf{v}_{N}^{k}}{\sqrt{c^{n}}}
$$

Thus, using the normalized sequency ordered eigenvectors, the eigenvalue decomposition (2) of the Hadamard matrix of order $N=2^{n}$, can be written as follows:

$$
\mathbf{H}_{N}=\mathbf{Z}_{N} \boldsymbol{\Lambda}_{N} \mathbf{Z}_{N}^{T}=\frac{1}{c^{n}} \mathbf{V}_{N} \boldsymbol{\Lambda}_{N} \mathbf{V}_{N}^{T}
$$

where $\boldsymbol{\Lambda}_{N}$ is the diagonal matrix whose non-zero elements are

$$
\lambda_{k}=(-1)^{k}=e^{-j k \pi}
$$

for $k=0,1, \ldots, N-1$. Hence, the definition (4) of the DFRHT matrix will take the form:

where

$$
\mathbf{H}_{N}^{a}=\frac{1}{c^{n}} \mathbf{V}_{N} \mathbf{\Lambda}_{N}^{a} \mathbf{V}_{N}^{T}
$$

$$
\lambda_{k}^{a}=e^{-j k \pi a}
$$

for $k=0,1, \ldots, N-1$. Based on the definition of the DFRHT matrix for $N=2^{n}$ it is easy to verify that the matrix has the unitary property [8]:

$$
\left(\mathbf{H}_{N}^{a}\right)^{-1}=\mathbf{H}_{N}^{-a}=\frac{1}{c^{n}} \mathbf{V}_{N} \boldsymbol{\Lambda}_{N}^{-a} \mathbf{V}_{N}^{T}
$$

\section{The algorithm}

Suppose you want to calculate the discrete fractional Hadamard transform for signal $\mathbf{x}_{N}$ in which the number of samples is equal to $N=2^{n}$. By $\mathbf{y}_{N}^{a}$ we denote the output signal which is calculated using the formula

$$
\mathbf{y}_{N}^{a}=\mathbf{H}_{N}^{a} \mathbf{x}_{N}
$$

In order to calculate the output signal it is necessary to perform $N^{2}$ complex multiplications and $N(N-1)$ complex additions, taking into account that the matrix $\mathbf{H}_{N}^{a}$ is given. If the input signal is real, then the number of real multiplications will be equal to $2 N^{2}$, and the number of real additions will be equal to $2 N(N-1)$. However, if 
we use the decomposition (14), and exploit the factorization of matrices $\mathbf{V}_{N}$ and $\mathbf{V}_{N}^{T}$ into a product of sparse matrices containing a small number of non-zero elements, the number of multiplications required to implement the DFRHT can be reduced. Factorization of the matrix is usually possible when it has a suitable structure. Let us consider the structure of matrices $\mathbf{V}_{N}$.

Example 2 The matrices $\mathbf{V}_{N}$ for $N=2,4,8$ are as follows:

$$
\begin{aligned}
& \mathbf{V}_{2}=\left[\begin{array}{cc}
1 & -b \\
b & 1
\end{array}\right], \quad \mathbf{V}_{4}=\left[\begin{array}{cccc}
1 & -b & b^{2} & -b \\
b & -b^{2} & -b & 1 \\
b & 1 & -b & -b^{2} \\
b^{2} & b & 1 & b
\end{array}\right] \\
& \mathbf{V}_{8}=\left[\begin{array}{cccccccc}
1 & -b & b^{2} & -b & b^{2} & -b^{3} & b^{2} & -b \\
b & -b^{2} & b^{3} & -b^{2} & -b & b^{2} & -b & 1 \\
b & -b^{2} & -b & 1 & -b & b^{2} & b^{3} & -b^{2} \\
b^{2} & -b^{3} & -b^{2} & b & 1 & -b & -b^{2} & b \\
b & 1 & -b & -b^{2} & b^{3} & b^{2} & -b & -b^{2} \\
b^{2} & b & -b^{2} & -b^{3} & -b^{2} & -b & 1 & b \\
b^{2} & b & 1 & b & -b^{2} & -b & -b^{2} & -b^{3} \\
b^{3} & b^{2} & b & b^{2} & b & 1 & b & b^{2}
\end{array}\right] .
\end{aligned}
$$

Example 2 demonstrates that the matrix $\mathbf{V}_{2}$ has a specific structure. As will be shown later, this type of matrices may be advantageously factorized. Now we consider the matrix $\mathbf{V}_{4}$. If the second and fourth columns of the matrix $\mathbf{V}_{4}$ are interchanged, and, the third and fourth columns of the resulting matrix are also interchanged, the following matrix will be obtained:

$$
\overline{\mathbf{V}}_{4}=\left[\begin{array}{cccc}
1 & -b & -b & b^{2} \\
b & 1 & -b^{2} & -b \\
b & -b^{2} & 1 & -b \\
b^{2} & b & b & 1
\end{array}\right]=\left[\begin{array}{cc}
\mathbf{V}_{2} & -b \mathbf{V}_{2} \\
b \mathbf{V}_{2} & \mathbf{V}_{2}
\end{array}\right]
$$

The matrix $\mathbf{V}_{4}$ differs from the matrix $\overline{\mathbf{V}}_{4}$ only in the order of the columns. Therefore, the matrix $\mathbf{V}_{4}$ can be obtained by postmultiplying the matrix $\overline{\mathbf{V}}_{4}$ by the permutation matrix $\mathbf{P}_{4}$, thus we can write:

$$
\mathbf{V}_{4}=\overline{\mathbf{V}}_{4} \mathbf{P}_{4}
$$

where:

$$
\mathbf{P}_{4}=\left[\begin{array}{llll}
1 & 0 & 0 & 0 \\
0 & 0 & 0 & 1 \\
0 & 1 & 0 & 0 \\
0 & 0 & 1 & 0
\end{array}\right]
$$

Now we consider the matrix $\mathbf{V}_{8}$. Let us perform the following permutation of columns of this matrix:

$$
\left(\begin{array}{llllllll}
1 & 2 & 3 & 4 & 5 & 6 & 7 & 8 \\
1 & 8 & 4 & 5 & 2 & 7 & 3 & 6
\end{array}\right) .
$$


As a result the following matrix is obtained:

$$
\overline{\mathbf{V}}_{8}=\left[\begin{array}{cccccccc}
1 & -b & -b & b^{2} & -b & b^{2} & b^{2} & -b^{3} \\
b & 1 & -b^{2} & -b & -b^{2} & -b & b^{3} & b^{2} \\
b & -b^{2} & 1 & -b & -b^{2} & b^{3} & -b & b^{2} \\
b^{2} & b & b & 1 & -b^{3} & -b^{2} & -b^{2} & -b \\
b & -b^{2} & -b^{2} & b^{3} & 1 & -b & -b & b^{2} \\
b^{2} & b & -b^{3} & -b^{2} & b & 1 & -b^{2} & -b \\
b^{2} & -b^{3} & b & -b^{2} & b & -b^{2} & 1 & -b \\
b^{3} & b^{2} & b^{2} & b & b^{2} & b & b & 1
\end{array}\right]=\left[\begin{array}{cc}
\overline{\mathbf{V}}_{4} & -b \overline{\mathbf{V}}_{4} \\
b \overline{\mathbf{V}}_{4} & \overline{\mathbf{V}}_{4}
\end{array}\right]
$$

As previously, we can write:

$$
\mathbf{V}_{8}=\overline{\mathbf{V}}_{8} \mathbf{P}_{8}
$$

where

$$
\mathbf{P}_{8}=\left[\begin{array}{llllllll}
1 & 0 & 0 & 0 & 0 & 0 & 0 & 0 \\
0 & 0 & 0 & 0 & 0 & 0 & 0 & 1 \\
0 & 0 & 0 & 1 & 0 & 0 & 0 & 0 \\
0 & 0 & 0 & 0 & 1 & 0 & 0 & 0 \\
0 & 1 & 0 & 0 & 0 & 0 & 0 & 0 \\
0 & 0 & 0 & 0 & 0 & 0 & 1 & 0 \\
0 & 0 & 1 & 0 & 0 & 0 & 0 & 0 \\
0 & 0 & 0 & 0 & 0 & 1 & 0 & 0
\end{array}\right]
$$

Generalizing the above mentioned considerations to the case $N=2^{n}$, we can write:

$$
\mathbf{V}_{N}=\overline{\mathbf{V}}_{N} \mathbf{P}_{N}
$$

for $N=2,4, \ldots, 2^{n}$. For $N=2$ we can also write

$$
\mathbf{V}_{2}=\overline{\mathbf{V}}_{2} \mathbf{P}_{2}=\overline{\mathbf{V}}_{2}
$$

where $\mathbf{P}_{2}$ is the identity matrix of order two

$$
\mathbf{P}_{2}=\mathbf{I}_{2} \text {. }
$$

The permutation matrix $\mathbf{P}_{N}$ of order $2^{n}$ can be obtained recursively from $\mathbf{P}_{N / 2}$ permutation matrix of order $2^{n-1}$ according to the following rule:

$$
\mathbf{P}_{2}=\left[\begin{array}{ll}
1 & 0 \\
0 & 1
\end{array}\right], \mathbf{P}_{N}=\mathbf{S}_{N}\left[\begin{array}{cc}
\mathbf{P}_{\frac{N}{2}} & \mathbf{0}_{\frac{N}{2}} \\
\mathbf{0}_{\frac{N}{2}} & \mathbf{P}_{\frac{N}{2}} \mathbf{J}_{\frac{N}{2}}
\end{array}\right]
$$

for $N=4,8, \ldots, 2^{n}$, where $\mathbf{S}_{N}$ is a perfect shuffle permutation matrix of order $2^{n}$, $\mathbf{J}_{N / 2}$ is a counter-identity matrix of order $N / 2$ and $\mathbf{0}_{N / 2}$ is zero matrix. The perfect 
shuffle permutation is the permutation that splits the set consisting of an even number of elements into two piles and interleaves them. It can be written in a following manner:

$$
\left(\begin{array}{cccccc}
1 & 2 & 3 & 4 & \ldots & 2 n \\
1 & n & 2 & n+1 & \ldots & 2 n
\end{array}\right)
$$

For example:

$$
\mathbf{S}_{8}=\left[\begin{array}{llllllll}
1 & 0 & 0 & 0 & 0 & 0 & 0 & 0 \\
0 & 0 & 0 & 0 & 1 & 0 & 0 & 0 \\
0 & 1 & 0 & 0 & 0 & 0 & 0 & 0 \\
0 & 0 & 0 & 0 & 0 & 1 & 0 & 0 \\
0 & 0 & 1 & 0 & 0 & 0 & 0 & 0 \\
0 & 0 & 0 & 0 & 0 & 0 & 1 & 0 \\
0 & 0 & 0 & 1 & 0 & 0 & 0 & 0 \\
0 & 0 & 0 & 0 & 0 & 0 & 0 & 1
\end{array}\right], \mathbf{J}_{4}=\left[\begin{array}{llll}
0 & 0 & 0 & 1 \\
0 & 0 & 1 & 0 \\
0 & 1 & 0 & 0 \\
1 & 0 & 0 & 0
\end{array}\right] .
$$

If we write the matrix $\mathbf{V}_{N}$ as a product $\overline{\mathbf{V}}_{N} \mathbf{P}_{N}$, and the multiplication by a factor responsible for the normalization of eigenvectors is included in the matrix of eigenvalues $\Lambda_{N}^{a}$, then the expression (14) will take the form:

$$
\mathbf{H}_{N}^{a}=\overline{\mathbf{V}}_{N} \mathbf{P}_{N} \tilde{\Lambda}_{N}^{a} \mathbf{P}_{N}^{T} \overline{\mathbf{V}}_{N}^{T}
$$

where

$$
\tilde{\Lambda}_{N}^{a}=\frac{1}{c^{n}} \Lambda_{N}^{a}
$$

and the matrix $\overline{\mathbf{V}}_{N}$ can be generated recursively:

$$
\overline{\mathbf{V}}_{2}=\left[\begin{array}{cc}
1 & -b \\
b & 1
\end{array}\right] \quad \overline{\mathbf{V}}_{2 k}=\left[\begin{array}{cc}
\overline{\mathbf{V}}_{k} & -b \overline{\mathbf{V}}_{k} \\
b \overline{\mathbf{V}}_{k} & \overline{\mathbf{V}}_{k}
\end{array}\right]
$$

for $k=2,4, \ldots, 2^{n-1}$. Such a construction of matrix $\overline{\mathbf{V}}_{N}$ enables a factorization, because we have the relationship

$$
\overline{\mathbf{V}}_{2 k}=\left[\begin{array}{cc}
\mathbf{I}_{k} & -b \mathbf{I}_{k} \\
b \mathbf{I}_{k} & \mathbf{I}_{k}
\end{array}\right]\left[\begin{array}{cc}
\overline{\mathbf{V}}_{k} & 0 \mathbf{I}_{k} \\
0 \mathbf{I}_{k} & \overline{\mathbf{V}}_{k}
\end{array}\right]
$$

where $\mathbf{I}_{k}$ is the identity matrix of order $k$.

It should be emphasized that the presence of permutation matrices $\mathbf{P}_{N}$ and $\mathbf{P}_{N}^{T}$ in expression (20) does not increase the number of arithmetic operations in the calculation of the DFRHT compared with the expression (14), since the multiplication by these matrices leads only to the permutation of the columns in the original matrix. 
Proposition 1 If the sequence of matrices $\left(\mathbf{W}_{2^{k}}\right)_{k=1,2, \ldots, n}$ is defined recursively

$$
\begin{gathered}
\mathbf{W}_{2}=\left[\begin{array}{cc}
p & q \\
r & s
\end{array}\right] \\
\mathbf{W}_{2^{k}}=\left[\begin{array}{cc}
p \mathbf{W}_{2^{k-1}} & q \mathbf{W}_{2^{k-1}} \\
r \mathbf{W}_{2^{k-1}} & s \mathbf{W}_{2^{k-1}}
\end{array}\right] \text { for } 2 \leq k \leq n
\end{gathered}
$$

then the matrix $\mathbf{W}_{2^{n}}$ is factorized as follows:

$$
\mathbf{W}_{2^{n}}=\mathbf{R}_{2^{n}}^{(n)} \mathbf{R}_{2^{n}}^{(n-1)} \ldots \mathbf{R}_{2^{n}}^{(1)}
$$

where $\left(\mathbf{R}_{2^{n}}^{(k)}\right)_{k=1,2, \ldots, n}$ is a quasi-diagonal matrix of order $2^{n}$ of the form

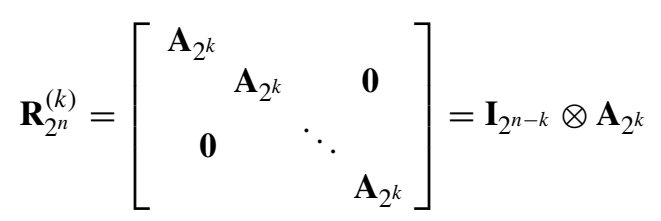

where the symbol $\otimes$ denotes the Kronecker product and sub-matrices $\mathbf{A}_{2^{k}}$, situated on the diagonal of the matrix $\mathbf{R}_{2^{n}}^{(k)}$, are given by:

$$
\mathbf{A}_{2^{k}}=\left[\begin{array}{cc}
p \mathbf{I}_{2^{k-1}} & q \mathbf{I}_{2^{k-1}} \\
r \mathbf{I}_{2^{k-1}} & s \mathbf{I}_{2^{k-1}}
\end{array}\right]=\mathbf{W}_{2} \otimes \mathbf{I}_{2^{k-1}}
$$

Proof Let $\left(\check{\mathbf{W}}_{2^{n}}^{(k)}\right)_{k=1,2, \ldots, n}$ be a sequence of quasi-diagonal matrices of the form:

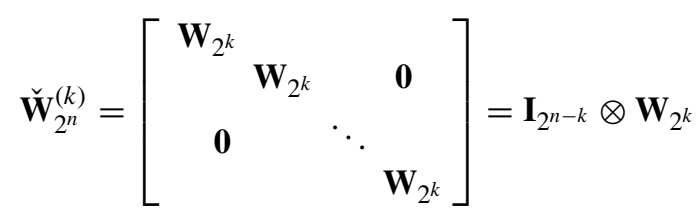

Note that there are equalities:

$$
\check{\mathbf{W}}_{2^{n}}^{(k+1)}=\mathbf{R}_{2^{n}}^{(k+1)} \mathbf{W}_{2^{n}}^{(k)}
$$

and

$$
\check{\mathbf{W}}_{2^{n}}^{(n)}=\mathbf{W}_{2^{n}}
$$

They result from the definition of the matrix $\mathbf{W}_{2^{n}}$ and from special constructions of matrices $\mathbf{R}_{2^{n}}^{(k)}$ and $\check{\mathbf{W}}_{2^{n}}^{(k)}$. Also the following decomposition occurs:

$$
\check{\mathbf{W}}_{2^{n}}^{(k)}=\mathbf{R}_{2^{n}}^{(k)} \mathbf{R}_{2^{n}}^{(k-1)} \ldots \mathbf{R}_{2^{n}}^{(1)} \text { for } 1 \leq k \leq n
$$

that can be justified by an induction method. 
For $k=1$ we get

$$
\check{\mathbf{W}}_{2^{n}}^{(1)}=\mathbf{R}_{2^{n}}^{(1)}=\left[\begin{array}{ccccccc}
p & q & & & & \\
r & s & & & & \mathbf{0} \\
& & p & q & & & \\
& & r & s & & & \\
& & & & \ddots & & \\
& & & & & p & q \\
& & & & & & \\
& & & & & s
\end{array}\right]=\mathbf{I}_{2^{n-1}} \otimes \mathbf{W}_{2} .
$$

Based on the induction hypothesis we have

$$
\check{\mathbf{W}}_{2^{n}}^{(k)}=\mathbf{R}_{2^{n}}^{(k)} \mathbf{R}_{2^{n}}^{(k-1)} \ldots \mathbf{R}_{2^{n}}^{(1)} .
$$

Thus, on the basis of (30) we obtain

$$
\mathbf{R}_{2^{n}}^{(k+1)}\left(\mathbf{R}_{2^{n}}^{(k)} \mathbf{R}_{2^{n}}^{(k-1)} \ldots \mathbf{R}_{2^{n}}^{(1)}\right)=\mathbf{R}_{2^{n}}^{(k+1)} \check{\mathbf{W}}_{2^{n}}^{(k)}=\check{\mathbf{W}}_{2^{n}}^{(k+1)}
$$

Taking in (32) $k=n$ we get $\check{\mathbf{W}}_{2^{n}}^{(n)}=\mathbf{R}_{2^{n}}^{(n)} \mathbf{R}_{2^{n}}^{(n-1)} \ldots \mathbf{R}_{2^{n}}^{(1)}$ and taking into account (31) we obtain the result.

For the matrix $\overline{\mathbf{V}}_{2^{n}}$ in (20), the conditions of Proposition 1 are satisfied when $p=s=1, q=-b$ and $r=b$. In this case,

$$
\mathbf{W}_{2}=\mathbf{V}_{2}=\left[\begin{array}{cc}
1 & -b \\
b & 1
\end{array}\right] \text {. }
$$

Hence, the matrix $\overline{\mathbf{V}}_{2^{n}}$ can be factorized as follows:

$$
\overline{\mathbf{V}}_{2^{n}}=\mathbf{R}_{2^{n}}^{(n)} \mathbf{R}_{2^{n}}^{(n-1)} \ldots \mathbf{R}_{2^{n}}^{(1)}
$$

where

$$
\mathbf{R}_{2^{n}}^{(k)}=\mathbf{I}_{2^{n-k}} \otimes \mathbf{V}_{2} \otimes \mathbf{I}_{2^{k-1}}
$$

for $k=0,1, \ldots, n$.

For the matrix $\overline{\mathbf{V}}_{2^{n}}^{T}$ in (20), the conditions of Proposition 1 are satisfied when $p=s=1, q=b$ and $r=-b$. In this case

$$
\mathbf{W}_{2}=\mathbf{V}_{2}^{T}=\left[\begin{array}{cc}
1 & b \\
-b & 1
\end{array}\right] .
$$

Then, the matrix $\overline{\mathbf{V}}_{2^{n}}^{T}$ can be factorized as follows:

$$
\overline{\mathbf{V}}_{2^{n}}^{T}=\tilde{\mathbf{R}}_{2^{n}}^{(n)} \tilde{\mathbf{R}}_{2^{n}}^{(n-1)} \ldots \tilde{\mathbf{R}}_{2^{n}}^{(1)}
$$

where

$$
\tilde{\mathbf{R}}_{2^{n}}^{(k)}=\mathbf{I}_{2^{n-k}} \otimes \mathbf{V}_{2}^{T} \otimes \mathbf{I}_{2^{k-1}}
$$

for $k=0,1, \ldots, n$.

Taking into account the factorization of the matrices $\overline{\mathbf{V}}_{2^{n}}$ and $\overline{\mathbf{V}}_{2^{n}}^{T}$ resulting from Proposition 1, a computational procedure that describes a fast discrete fractional 
Hadamard transform algorithm for the vector $\mathbf{x}_{N}$ of length $N=2^{n}$ can be represented as follows:

$$
\mathbf{y}_{N}^{a}=\mathbf{R}_{N}^{(n)} \mathbf{R}_{N}^{(n-1)} \ldots \mathbf{R}_{N}^{(1)} \mathbf{P}_{N} \tilde{\Lambda}_{N}^{a} \mathbf{P}_{N}^{T} \tilde{\mathbf{R}}_{N}^{(n)} \tilde{\mathbf{R}}_{N}^{(n-1)} \ldots \tilde{\mathbf{R}}_{N}^{(1)} \mathbf{x}_{N}
$$

The explicit form of the matrices occurring in the expression (37) and the flow diagram for the DFRHT for $N=8$ are presented in the Appendix. In the proposed algorithm the matrix-vector products $\tilde{\mathbf{R}}_{N}^{(k)} \mathbf{x}_{N}^{k-1}$, where $\mathbf{x}_{N}^{0}$ is the input vector, are calculated step by step. Actually the multiplications of the matrix $\mathbf{V}_{2}$ by the two-element sub-vectors of the vector $\mathbf{x}_{N}^{k-1}$ are implemented. Each of this products requires 2 real multiplications and 2 real additions. Since the matrix $\mathbf{V}_{2}$ is not orthogonal $\left(\mathbf{V}_{2} \mathbf{V}_{2}^{T}=c \mathbf{I}_{2}\right)$, repeatedly applying this matrix can be a source of rounding errors. To avoid this problem the matrix $\mathbf{V}_{2}$ can be replaced by the matrix $\frac{1}{\sqrt{c}} \mathbf{V}_{2}$, which is orthogonal, hence numerically stable. In this case, the matrices $\tilde{\mathbf{R}}_{N}^{(k)}$ and $\mathbf{R}_{N}^{(k)}$, occurring in our algorithm, should by replaced by the orthogonal matrices $\frac{1}{\sqrt{c}} \tilde{\mathbf{R}}_{N}^{(k)}$ and $\frac{1}{\sqrt{c}} \mathbf{R}_{N}^{(k)}$ respectively, and the matrix $\tilde{\Lambda}_{N}^{a}$ by the unitary matrix $\Lambda_{N}^{a}$. After such replacement the unitary matrix $\mathbf{H}_{N}^{a}$ would be factorized as a product of unitary factors. However, such solution leads to a doubling of the number of multiplications in comparison with our algorithm, since the two entries equal to 1 in $\mathbf{V}_{2}$ are replaced by $1 / \sqrt{c}$ in $\frac{1}{\sqrt{c}} \mathbf{V}_{2}$, so we will try to estimate how big is the potential loss of accuracy in our algorithm. For $N=2^{n}$, the unitary alternative means dividing $n$ times by $\sqrt{c} \approx 1.0824$ then the diagonal means multiplying with $c^{n}$ and then again $n$ divisions by $\sqrt{c}$. For example, if $N=2^{50} \approx 10^{15}$, we divide by $c^{n / 2} \approx 52$, multiply by $c^{n} \approx 2744$ and divide again by $c^{n / 2} \approx 52$. Since this means a potential loss of only 2 to 3 decimal digits for such a large $N$ that in practice, using our fast, non-unitary version, does not seem to be a serious problem.

Since the DFRHT matrix satisfies property (16), the inverse discrete fractional Hadamard transform (IDFRHT) can be calculated in the same manner as the DFRHT transform, only the parameter $a$ should be changed to $-a$. The general procedure for calculating the IDFRHT transform for $N=2^{n}$ will take the form

$$
\mathbf{x}_{N}=\mathbf{R}_{N}^{(n)} \mathbf{R}_{N}^{(n-1)} \ldots \mathbf{R}_{N}^{(1)} \mathbf{P}_{N} \tilde{\Lambda}_{N}^{-a} \mathbf{P}_{N}^{T} \tilde{\mathbf{R}}_{N}^{(n)} \tilde{\mathbf{R}}_{N}^{(n-1)} \ldots \tilde{\mathbf{R}}_{N}^{(1)} \mathbf{y}_{N}^{a}
$$

\section{Computational complexity}

Let us assess the computational complexity in terms of number multiplications and additions required for the DFRHT calculation. Calculation of the discrete fractional Hadamard transform for a real-valued vector $\mathbf{x}_{N}$ of length $N=2^{n}$, assuming that the matrix $\mathbf{H}_{N}^{a}$ defined by (4) is given, requires $N^{2}=2^{2 n}$ multiplications of a complex number by a real number and $N(N-1)=2^{n}\left(2^{n}-1\right)$ complex additions. Each multiplication of a complex number by a real number needs two real multiplications and each addition of two complex numbers requires two real additions. Hence, the number of real multiplications and real additions required for computing 
the DFRHT using the direct method is equal to $2^{2 n+1}$ and $2^{n+1}\left(2^{n}-1\right)$, respectively, thus computational complexity of the direct method is $O\left(N^{2}\right)$.

Proposition 2 The computational complexity of the DFRHT calculated according to our fast algorithm is $\mathrm{O}\left(\mathrm{N} \log _{2} N\right)$.

Proof A real-valued matrix-vector product $\tilde{\mathbf{R}}_{N}^{(k)} \mathbf{x}_{N}$ in formula (37) requires $2^{n}$ real multiplications and $2^{n}$ real additions, so calculation of the product $\tilde{\mathbf{R}}_{N}^{(n)} \tilde{\mathbf{R}}_{N}^{(n-1)} \ldots \tilde{\mathbf{R}}_{N}^{(1)} \mathbf{x}_{N}$ required $n 2^{n}$ real multiplications and $n 2^{n}$ real additions. As a result, we again obtain a real-valued vector. As it was already mentioned, the multiplication of the permutation matrix $\mathbf{P}_{N}^{T}$ by a vector does not require any arithmetic operations. The product of the complex-valued diagonal matrix $\tilde{\Lambda}_{N}^{a}$ (we assume that for a predetermined parameter $a$, the diagonal elements of this matrix were calculated in advance) and real-valued vector (alredy calculated) requires $2^{n+1}-1$ real multiplications (the first entry $\tilde{\lambda}_{0}^{a}$ is always real). The resulting product is complexvalued. Applying the permutation $\mathbf{P}_{N}$ does not require any arithmetical operations. The resulting vector is then multiplied by the matrices $\mathbf{R}_{N}^{(1)}, \mathbf{R}_{N}^{(2)}, \ldots, \mathbf{R}_{N}^{(n)}$. Each of these multiplications requires $2^{n+1}$ real multiplications and $2^{n+1}$ real additions, so all together they require $n 2^{n+1}$ real multiplications and $n 2^{n+1}$ real additions. The total number of arithmetic operations to compute DFRHT of size $2^{n}$ using our fast algorithm is $(3 n+2) 2^{n}-1$ real multiplications and $3 n 2^{n}$ real additions, so the computational complexity of this algorithm is $\mathrm{O}\left(\mathrm{N} \log _{2} \mathrm{~N}\right)$.

Tables 1 and 2 display the number of multiplications and additions required for the DFRHT transform implementation of the real-valued input signal of the length $N=2^{n}$. These numbers were calculated for three methods of the transform implementation: the direct multiplication of the DFRHT matrix by a vector of the input data, calculation according to authors' algorithm described in the work [9], and according to the algorithm (37) proposed in this article. It is easy to check that even

Table 1 Number of multiplications for mentioned algorithms

\begin{tabular}{llll}
\hline$N=2^{n}$ & Direct method & Method [9] & Proposed algorithm \\
\hline 2 & 8 & 6 & 9 \\
4 & 32 & 18 & 31 \\
8 & 128 & 54 & 87 \\
16 & 512 & 162 & 223 \\
32 & 2048 & 486 & 543 \\
64 & 8192 & 1458 & 1279 \\
128 & 32768 & 4374 & 2943 \\
256 & 131072 & 13122 & 6655 \\
512 & 524288 & 39366 & 14847 \\
1024 & 2097152 & 118098 & 32767 \\
\hline
\end{tabular}


Table 2 Number of additions for mentioned algorithms

\begin{tabular}{llll}
\hline$N=2^{n}$ & Direct method & Method [9] & Proposed algorithm \\
\hline 2 & 4 & 5 & 6 \\
4 & 24 & 25 & 24 \\
8 & 112 & 95 & 72 \\
16 & 480 & 325 & 192 \\
32 & 1984 & 1055 & 480 \\
64 & 8064 & 3325 & 1152 \\
128 & 32512 & 10295 & 2688 \\
256 & 130560 & 31525 & 6144 \\
512 & 523264 & 95855 & 13824 \\
1024 & 2095104 & 290125 & 30720 \\
\hline
\end{tabular}

for not very large $n$ the number of arithmetic operations, required for DFRHT transform realization according to the proposed algorithm, is several times smaller than the other two methods of DFRHT computing.

\section{Conclusions}

The article presents a fast algorithm for the DFRHT performing. The algorithm has a much lower computational complexity than the direct way of the DFRHT implementation. Although the authors' paper [9] describes a rationalized algorithm for the DFRHT with a reduced number of multiplications and additions, the solution presented in that article does not exhaust all possibilities of rationalizing the process of the DFRHT implementation. The algorithm presented in this article has a lower computational complexity than the algorithm in paper [9], especially in the case of large values of $N$.

In this article the computational procedure for DFRHT calculating is described in matrix notation. The matrix notation is a very compact and simple mathematical formalism suitable for parallel realization. This notation enables us to adequately represent the space-time structures of an implemented computational process and directly maps these structures into the hardware realization space. For simplicity, we considered the synthesis of a fast algorithm for the DFRHT calculation for $N=2^{3}$ (in Appendix). However, it is clear that the proposed procedure was developed for an arbitrary case when the order of the matrix is a power of two.

Acknowledgments The authors would like to thank the anonymous reviewers for their great effort and valuable comments that helped to improve the clarity and quality of this manuscript. 


\section{Appendix}

For $N=8$ the fast algorithm (37) for computing the discrete fractional Hadamard transform will be represented by the following vector-matrix procedure:

$$
\mathbf{y}_{8}^{a}=\mathbf{R}_{8}^{(3)} \mathbf{R}_{8}^{(2)} \mathbf{R}_{8}^{(1)} \mathbf{P}_{8} \tilde{\Lambda}_{8}^{a} \mathbf{P}_{8}^{T} \tilde{\mathbf{R}}_{8}^{(3)} \tilde{\mathbf{R}}_{8}^{(2)} \tilde{\mathbf{R}}_{8}^{(1)} \mathbf{x}_{8},
$$

where the matrix $\mathbf{P}_{8}$ was previously determined and the other matrices take the following form:

$$
\begin{aligned}
& \mathbf{R}_{8}^{(3)}=\mathbf{I}_{1} \otimes \mathbf{V}_{2} \otimes \mathbf{I}_{4}=\left[\begin{array}{cccccccc}
1 & 0 & 0 & 0 & -b & 0 & 0 & 0 \\
0 & 1 & 0 & 0 & 0 & -b & 0 & 0 \\
0 & 0 & 1 & 0 & 0 & 0 & -b & 0 \\
0 & 0 & 0 & 1 & 0 & 0 & 0 & -b \\
b & 0 & 0 & 0 & 1 & 0 & 0 & 0 \\
0 & b & 0 & 0 & 0 & 1 & 0 & 0 \\
0 & 0 & b & 0 & 0 & 0 & 1 & 0 \\
0 & 0 & 0 & b & 0 & 0 & 0 & 1
\end{array}\right], \\
& \mathbf{R}_{8}^{(2)}=\mathbf{I}_{2} \otimes \mathbf{V}_{2} \otimes \mathbf{I}_{2}=\left[\begin{array}{cccccccc}
1 & 0 & -b & 0 & 0 & 0 & 0 & 0 \\
0 & 1 & 0 & -b & 0 & 0 & 0 & 0 \\
b & 0 & 1 & 0 & 0 & 0 & 0 & 0 \\
0 & b & 0 & 1 & 0 & 0 & 0 & 0 \\
0 & 0 & 0 & 0 & 1 & 0 & -b & 0 \\
0 & 0 & 0 & 0 & 0 & 1 & 0 & -b \\
0 & 0 & 0 & 0 & b & 0 & 1 & 0 \\
0 & 0 & 0 & 0 & 0 & b & 0 & 1
\end{array}\right], \\
& \mathbf{R}_{8}^{(1)}=\mathbf{I}_{4} \otimes \mathbf{V}_{2} \otimes \mathbf{I}_{1}=\left[\begin{array}{cccccccc}
1 & -b & 0 & 0 & 0 & 0 & 0 & 0 \\
b & 1 & 0 & 0 & 0 & 0 & 0 & 0 \\
0 & 0 & 1 & -b & 0 & 0 & 0 & 0 \\
0 & 0 & b & 1 & 0 & 0 & 0 & 0 \\
0 & 0 & 0 & 0 & 1 & -b & 0 & 0 \\
0 & 0 & 0 & 0 & b & 1 & 0 & 0 \\
0 & 0 & 0 & 0 & 0 & 0 & 1 & -b \\
0 & 0 & 0 & 0 & 0 & 0 & b & 1
\end{array}\right], \\
& \tilde{\mathbf{R}}_{8}^{(3)}=\mathbf{I}_{1} \otimes \mathbf{V}_{2}^{T} \otimes \mathbf{I}_{4}=\left[\begin{array}{cccccccc}
1 & 0 & 0 & 0 & b & 0 & 0 & 0 \\
0 & 1 & 0 & 0 & 0 & b & 0 & 0 \\
0 & 0 & 1 & 0 & 0 & 0 & b & 0 \\
0 & 0 & 0 & 1 & 0 & 0 & 0 & b \\
-b & 0 & 0 & 0 & 1 & 0 & 0 & 0 \\
0 & -b & 0 & 0 & 0 & 1 & 0 & 0 \\
0 & 0 & -b & 0 & 0 & 0 & 1 & 0 \\
0 & 0 & 0 & -b & 0 & 0 & 0 & 1
\end{array}\right],
\end{aligned}
$$




$$
\begin{gathered}
\tilde{\mathbf{R}}_{8}^{(2)}=\mathbf{I}_{2} \otimes \mathbf{V}_{2}^{T} \otimes \mathbf{I}_{2}=\left[\begin{array}{cccccccc}
1 & 0 & b & 0 & 0 & 0 & 0 & 0 \\
0 & 1 & 0 & b & 0 & 0 & 0 & 0 \\
-b & 0 & 1 & 0 & 0 & 0 & 0 & 0 \\
0 & -b & 0 & 1 & 0 & 0 & 0 & 0 \\
0 & 0 & 0 & 0 & 1 & 0 & b & 0 \\
0 & 0 & 0 & 0 & 0 & 1 & 0 & b \\
0 & 0 & 0 & 0 & -b & 0 & 1 & 0 \\
0 & 0 & 0 & 0 & 0 & -b & 0 & 1
\end{array}\right], \\
\tilde{\mathbf{R}}_{8}^{(1)}=\mathbf{I}_{4} \otimes \mathbf{V}_{2}^{T} \otimes \mathbf{I}_{1}=\left[\begin{array}{cccccccc}
1 & b & 0 & 0 & 0 & 0 & 0 & 0 \\
-b & 1 & 0 & 0 & 0 & 0 & 0 & 0 \\
0 & 0 & 1 & b & 0 & 0 & 0 & 0 \\
0 & a & -b & 1 & 0 & 0 & 0 & 0 \\
0 & 0 & 0 & 0 & 1 & b & 0 & 0 \\
0 & 0 & 0 & 0 & -b & 1 & 0 & 0 \\
0 & 0 & 0 & 0 & 0 & 0 & 1 & b \\
0 & 0 & 0 & 0 & 0 & 0 & -b & 1
\end{array}\right], \\
\tilde{\Lambda}_{8}^{a}=\frac{1}{c^{3}}\left[\begin{array}{cccccccccc}
\lambda_{0}^{a} & 0 & 0 & 0 & 0 & 0 & 0 & 0 \\
0 & \lambda_{1}^{a} & 0 & 0 & 0 & 0 & 0 & 0 \\
0 & 0 & \lambda_{2}^{a} & 0 & 0 & 0 & 0 & 0 \\
0 & 0 & 0 & \lambda_{3}^{a} & 0 & 0 & 0 & 0 \\
0 & 0 & 0 & 0 & \lambda_{4}^{a} & 0 & 0 & 0 \\
0 & 0 & 0 & 0 & 0 & \lambda_{5}^{a} & 0 & 0 \\
0 & 0 & 0 & 0 & 0 & 0 & \lambda_{6}^{a} & 0 & \\
0 & 0 & 0 & 0 & 0 & 0 & 0 & \lambda_{7}^{a}
\end{array}\right]
\end{gathered}
$$

where $c=1+(\sqrt{2}-1)^{2}, \lambda_{0}^{a}=e^{-0 j \pi a}=1, \lambda_{1}^{a}=e^{-1 j \pi a}, \lambda_{2}^{a}=e^{-2 j \pi a}, \lambda_{3}^{a}=$ $e^{-3 j \pi a}, \lambda_{4}^{a}=e^{-4 j \pi a}, \lambda_{5}^{a}=e^{-5 j \pi a}, \lambda_{6}^{a}=e^{-6 j \pi a}, \lambda_{7}^{a}=e^{-7 j \pi a}$.

Figure 1 shows a data flow diagram of the fast algorithm for the 8-point DFRHT. In this paper, the data flow diagram is oriented from left to right. Straight lines in the

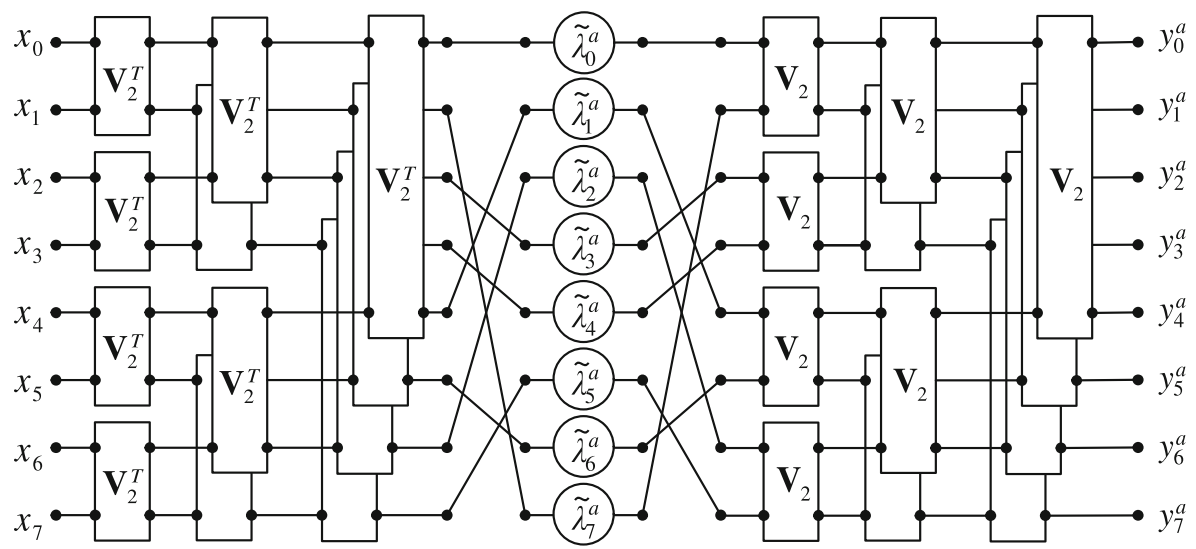

Fig. 1 Data flow diagram of the fast DFRHT algorithm for $N=8$ 
figures denote the operation of data transfer. We use the usual lines without arrows specifically not to clutter the picture. Note that the circles in this figure shows the operations of multiplication by a complex number inscribed inside a circle. In turn, the rectangles indicate the matrix-vector multiplications by matrices $\mathbf{V}_{2}$ and $\mathbf{V}_{2}^{T}$.

\section{References}

1. Yetik, I.Ş., Kutay, M.A., Ozaktas, H.M.: Image representation and compression with the fractional Fourier transform. Opt. Commun. 197, 275-278 (2001)

2. Hennelly, B., Sheridan, J.T.: Fractional Fourier transform-based image encryption: phase retrieval algorithm. Opt. Commun. 226, 61-80 (2003)

3. Liu, S., Mi, Q., Zhu, B.: Optical image encryption with multistage and multichannel fractional Fourierdomain filtering. Opt. Lett. 26, 1242-1244 (2001)

4. Nischchal, N.K., Joseph, J., Singh, K.: Fully phase encryption using fractional Fourier transform. Opt. Eng. 42, 1583-1588 (2003)

5. Djurović, I., Stanković, S., Pitas, I.: Digital watermarking in the fractional Fourier transformation domain. J. Netw. Comput. Appl. 24, 167-173 (2001)

6. Pei, S.C., Yeh, M.H.: The discrete fractional cosine and sine transforms. IEEE Trans. Sig. Proc. 49, 1198-1207 (2001)

7. Pei, S.C., Tseng, C.C., Yeh, M.H., Shyu, J.J.: The discrete fractional Hartley and Fourier transforms. IEEE Trans. Circuits Syst. Part II 45, 665-675 (1998)

8. Pei, S.C., Yeh, M.H., Shyu, J.J.: Discrete fractional Hadamard transform, Vol. 3, pp. 179-182 (1999)

9. Majorkowska-Mech, D., Cariow, A.: An algorithm for discrete fractional Hadamad transform with reduced arithmetical complexity. Przeglad Elektrotechniczny (Electrical Review) 88, 70-76 (2012)

10. Sylvester, J.J.: Thoughts on inverse orthogonal matrices, simultaneous sign-successions, and tessellated pavements in two or more colours, with applications to Newton's rule, ornamental tile-work, and the theory of numbers. Philos. Mag. 34, 461-475 (1867)

11. Bella, T., Olshevsky, V., Sankhnovich L: Ranks of Hadamard matrices and equivalence of Sylvester Hadamard and pseudo-noise matrices. Operator Theory: Advances and Applications, Vol. 179, pp. 3546. Birkhauser, Verlag, Basel/Switzerland (2007)

12. Mitrouli, M.: A sign test for detecting the equivalence of Sylvester Hadamard matrices. Numer. Algor. 57, 169-186 (2011)

13. Horadam, K.J.: Hadamard Matrices and their Applications. Princeton University Press, Princeton (2006)

14. Sloane, N.J.A., MacWilliams F.J.: The Theory of Error-Correcting Codes. Elsevier/North-Holland, Amsterdam (1977)

15. Korn, G.A., Korn, T.M.: Mathematical Handbook for Scientists and Engineers: Definitions, Theorems, and Formulas for Reference and Review. McGraw-Hill, New York (1968)

16. Candan, Ç.C., Kutay, M.A., Ozaktas, H.M.: The discrete fractional Fourier transform. IEEE Trans. Sig. Proc. 48, 1329-1337 (2000)

17. Yarlagadda, R., Hershey, J.: A note on the eigenvectors of Hadamard matrices of order $2^{n}$. Linear Algebra Appl. 45, 43-53 (1982)

18. Yarlagadda, R.K.R., Hershey, J.E.: Hadamard Matrix Analysis and Synthesis. Kluwer Academic Publishers, Boston (1997) 